Págs. 239-257

\title{
El «paradigma biográfico» en la investigación educativa
} The «biographical paradigm» in educational research

\section{Peter Alheit ${ }^{1}$}

\section{Resumen}

El artículo muestra el amplio rango de las concepciones que cubren las concepciones formativas orientadas biográficamente: la amplitud de los planteamientos va desde intervenciones terapéuticas individuales hasta el compromiso sociopolítico, desde temas clásicos de la formación continua de índole general y sociocultural hasta las nuevas cuestiones de formación continua profesional y política. Por lo tanto, la orientación de la biografía en la formación de personas (adultas) puede considerarse como una especie de «paradigma oculto» que, además, sugiere un cambio gradual en las disposiciones de los profesionales.

\section{Palabras clave}

Biograficidad, paradigma biográfico, educación, curso de vida.

\section{Abstract}

The article shows the wide range of conceptions that cover the biographical orientated formative conceptions: the amplitude of the expositions goes from individual therapeutic interventions to the sociopolitical compromise, from classic subjects of the continuous training of general and sociocultural nature to the new questions of continuous, professional, and political formation. Therefore, the orientation of biography in the formation of people (adults) can be considered as a kind of «hidden paradigm» that, in addition, suggests a gradual change in the dispositions of professionals.

\section{Keywords}

Biograficity, biographical paradigm, education, life course. 


\section{Introducción}

Las biografías se pueden interpretar, por un lado, como ordenaciones secuenciales de patrones predefinidos socialmente, que no se pueden cambiar arbitrariamente. Aquí radica el aspecto estructural de lo biográfico. Por ello, el constructo plausible de una «institucionalización» que tenga lugar en el proceso de la modernidad tiene probablemente que ser precisado todavía con vistas al proceso de erosión actual ${ }^{2}$. También la elaboración de estructuras biográficas con un alcance menor, como las «estructuras procesuales del curso de vida» de Schütze siguen siendo un desideratum significativo. Por otro lado, las biografías no se pueden aprehender si se ignora la dimensión de la acción emergente, del empeño ${ }^{3}$, lo individual en el proceso biográfico.

Sin embargo, esta doble perspectiva no se tiene que presentar como una interacción armónica de dos planos: la dimensión de la acción emergente y la dimensión de la estructura, la perspectiva del objeto y la del sujeto, que no «encajan» inmediatamente una con otra. Entre ellas existe una tensión dialéctica que, en primer lugar, permite la subjetividad. Una acción biográfica se orienta según patrones que presentan cursos prescritos socialmente, está señalizada por ellos y no puede escapar a sus coerciones, y no solo en su ejecución posterior. No reproduce meramente determinadas condiciones estructurales sociales en el plano individual, «sino que tiene siempre también el carácter de un esbozo abierto» (Kohli, 1985: 21). Las experiencias y los patrones de acción que se adquieren en el curso de una biografía no se acumulan simplemente de un modo cuantitativo. Hay saltos cualitativos, rupturas, replanteamientos sorprendentes, momentos de acción emergente y autonomía. Y precisamente esta ambigüedad hace que el fenómeno social biografía sea, desde la perspectiva de la estrategia conceptual, tan interesante. No solo el rendimiento teórico de la sociología o de la pedagogía (como, por ejemplo, en los casos de los diferentes conceptos de identidad o de socialización), sino la experiencia vivida de lo biográfico mismo crea la tensión productiva de la perspectiva del objeto y la del sujeto, que es importante para las cuestiones pedagógicas.

De un modo asombroso, esta dialéctica se encuentra ya en un texto clásico de la pedagogía. Friedrich Schleiermacher, en sus Lecciones de 1826, determina como la meta de la educación una tarea doble: «a saber, capacitar para la comunidad y el desarrollo de la peculiaridad personal» (Schleiermacher, 1957: 66) -en expresión más moderna: ejercitarse en las estructuras y la apertura al curso de vida personal-. En un sentido muy abstracto, esta doble opción es ciertamente una trivialidad de la teorización pedagógica. Si se siguen, por el contrario, las tradiciones históricas de las ciencias de la educación (no solo en Alemania) con pretensión crítica, entonces resulta claro que esta dialéctica no sirvió de ninguna manera a una formación conceptual. Las teorías pedagógicas clásicas hipostasiaron no solo el despliegue del individuo desprendido de las condiciones estructurales y allanaron el camino para el «desarrollo de la peculiaridad personal», en el mejor de los casos para los socialmente privilegiados ${ }^{4}$. El «empoderamiento de la comunidad» al precio de la peculiaridad personal ha producido en los dos últimos siglos una serie de ejemplos disuasivos (cf. como ejemplo Gamm 1984). E incluso la defensa del sujeto contra su instrumentalización, reclamada en las teorías críticas de la formación con conceptos como «emancipación», «responsabilidad»

2 Tenemos que preguntarnos si, en este punto, el modelo «institucional» sigue siendo todavía el más certero o si no resultarán más adecuados otros, como, por ejemplo, el concepto de Glen Elder de «transition» (Elder, 1985).

3 Alheit utiliza la palabra Eigensinn, que juega un papel importante en su teoría y en las elaboraciones de Bettina Dausien, y no tiene un equivalente exacto en castellano. La palabra alemana se forma con Sinn «sentido» y eigen «propio»; literalmente es «sentido propio». Se suele traducir al castellano por «obstinación», que procede del latín obstantio, que sería la acción de «estar de pie, frente a algo», contra lo cual se persevera con terquedad. Pero «obstinación» o «terquedad» tienen un significado negativo ausente en la etimología alemana, por lo que hemos preferido el término «empeño». La construcción de toda biografía y cualquier proceso formativo requieren que el sujeto se empeñe, que persevere en su sentido propio, el del sujeto. (N. trad.)

4 Véase la adecuada crítica de Theodor Schulze (1985: 29 y s.). 
o «autodeterminación», mantiene una distancia voluntaria con los individuos concretos (cf. Schulze, 1985: $30)$.

La importancia reciente del término «biografía», también en los contextos de las ciencias de la educación, sin embargo, parece tener que ver menos con la reflexión crítica sobre las tradiciones de la teoría de la educación de los últimos dos siglos, que con fenómenos sintomáticos de la época: los individuos tienen que producir hoy en día -evidentemente más que nunca- el equilibrio entre las exigencias objetivas y las peculiaridades subjetivas. El «empoderamiento de la comunidad» y el «desarrollo de la peculiaridad individual» ya no resultan apenas integrables. En este punto, la biografía misma se pone en el foco de atención. Los portadores de las biografías «deben aprender, bajo pena de ruptura de su personalidad o de un prejuicio social permanente, a vincular por ellos mismos diferentes campos de experiencia y de acción [...] Deben igualmente saber equilibrar por ellos mismos las demandas y las exigencias aparentemente incompatibles de espacios sociales, de instituciones y dominios de la vida múltiples y contradictorios entre ellos, a fin de poder vivirlos en lo cotidiano. Los imperativos de la integración social refuerzan aún más esta tendencia: es a los individuos en cuanto tales, y no a los grupos sociales primarios, a los que compete la tarea de vincular y coordinar sus acciones y aquello que pueden pretender en su vida [...] $\mathrm{O}$ los individuos crean por ellos mismos la socialidad o resultan amenazados de quedar al margen y de aislamiento social». (Körber, 1989: 139).

Este inusualmente agudo diagnóstico de la época lleva a consecuencias respecto de la teoría de la formación. Con la «tesis de la individualización» (Beck, 1986: 205 y ss.) $)^{5}$-por cierto, bastante controvertida desde un punto de vista empírico- ha obtenido también una etiqueta de efecto publicitario. Parece plausible vincular la importancia creciente del tema biográfico en la pedagogía con este importante diagnóstico de la actualidad. Sin duda, este síntoma de la época no garantiza ninguna teoría convincente. Y, de hecho, no generó un concepto consistente de «pedagogía biográfica», aunque sí una serie de planteamientos más o menos elaborados, que vale la pena considerar con más detalle. En aras de la claridad, a continuación se distinguirán y se analizarán críticamente seis planteamientos ${ }^{6}$ :

- El «planteamiento antropológico»,

- El «planteamiento compensatorio»,

- El «planteamiento autobiográfico»,

- El «planteamiento histórico»,

- El «planteamiento intercultural»,

- El «planteamiento emancipatorio».

\section{El «planteamiento antropológico»}

Con esta etiqueta se vincula la «teoría educativa biográfica» más elaborada y más establecida en el ámbito lingüístico alemán, la concepción de Werner Loch y sus discípulos (resulta representativo Spanhel (ed.)

5 En el ámbito macrosociológico, esta tesis todavía parece extraordinariamente especulativa. La «índole estructural de la desigualdad social»-la efectividad del estrato, la clase, la adscripción étnica, el género, etc.- (Kohli, 1989: 266) no ha retrocedido significativamente. Por el contrario, hay modificaciones incuestionables en el ámbito microsocial.

6 Ciertamente, esta diferenciación no es arbitraria e incluso las denominaciones elegidas tienen una gran afinidad con la manera como se presentan a sí mismos los planteamientos concretos, que incluso se presentan con una pretensión sistemática y siguen una cierta «dramaturgia» en sus modos de exposición. Esto significa que la diferenciación ni reclama completud, ni excluye una sistemática que se puede contrastar. 
1988). Loch enlazó en su obra principal Lebenslauf und Eræ̌iebung [Curso de vida y educación] (1979) con la tradición de las pedagogía de las ciencias del espíritu, en particular con Dilthey (Loch, 1979: 121 y ss.) y amplió el vitalismo pedagógico en aspectos fenomenológicos y de teoría del lenguaje (Loch, 1981: 37 y ss.). La intención de este diseño teórico es demostrar «que el individuo humano que ha llegado al mundo tiene que desarrollar en el curso de su vida una serie de capacidades, y normalmente puede desarrollarlas, que se pueden exponer como una serie significativa y necesariamente antropológica de etapa de capacidad» (Loch, 1981: 33). Loch caracteriza estas capacidades, que recuerdan ${ }^{7}$ un teorema clásico de etapas de la pedagogía y la psicología del desarrollo, como «competencias curriculares». Sin embargo, el concepto curricular tiene aquí poco en común con los modelos tecnocráticos del currículum. Más bien se refiere explícitamente a aquel curriculum vitae que ya en Comenio debía de garantizar la completud del proceso educativo.

Loch adopta una «serie de etapas de habilidades» que cada ser humano tiene que atravesar para poder superar determinadas «situaciones curriculares» (es decir, situaciones del curso de la vida) (Loch, 1981: 41 y ss.). En el caso de una sincronización deficiente de las competencias curriculares y las situaciones curriculares, se producen «entorpecimientos del aprendizaje». En este punto, la educación se vuelve relevante. Ella intenta, comenzando con la atención materna, contrarrestar el entorpecimiento del aprendizaje con una «ayuda para el aprendizaje». En el transcurso de la biografía pueden darse «conflictos curriculares» muy diversos, incluso fricciones entre competencias y situaciones, a lo que reaccionan los denominados «patrones curriculares de educación». En este proceso puede desplegarse aquel esquema que Loch, en su «modelo egológico de educación», denomina la «cruz de la educación» (1981: 46):

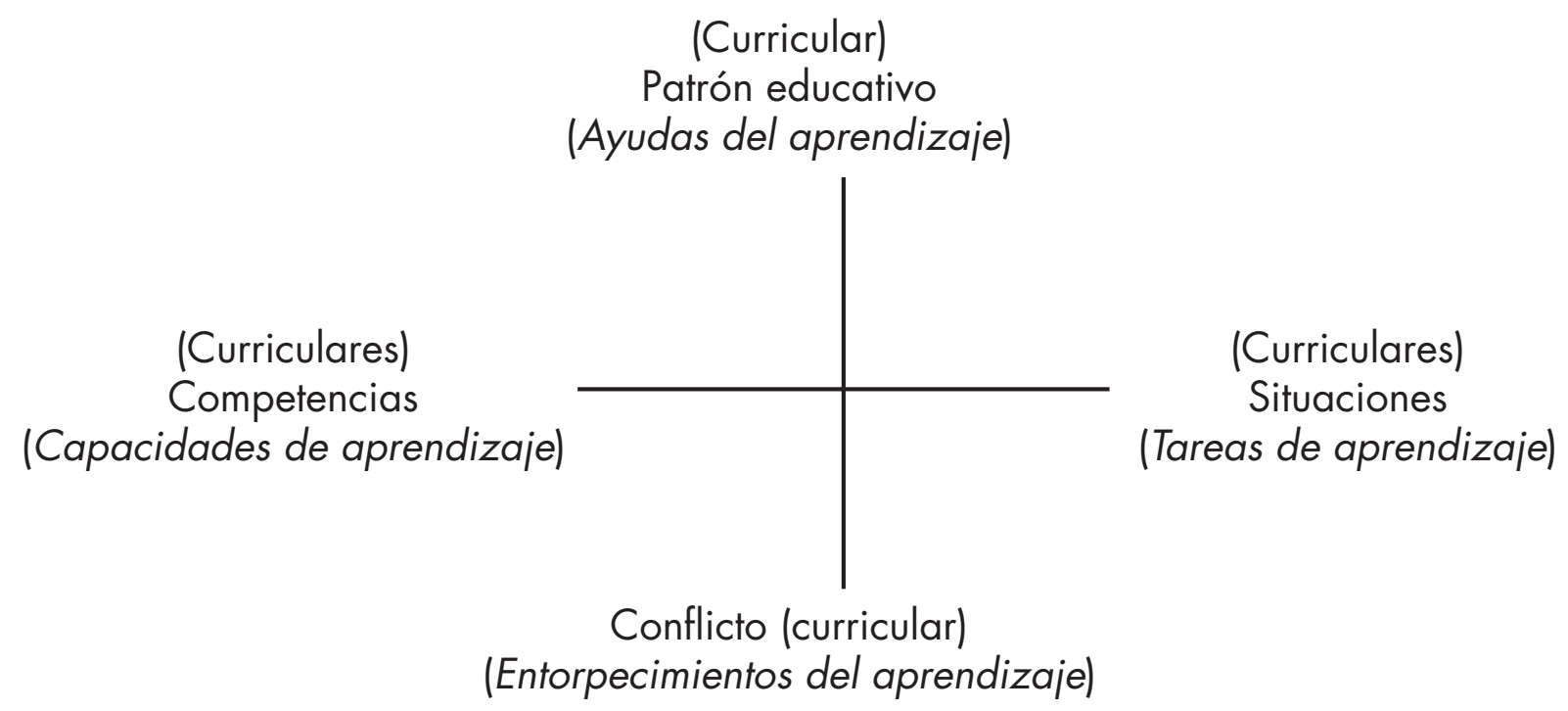

La claridad de este esquema también indica sus limitaciones. La consistencia de la teoría de la educación biográfica de Loch se debe al recurso ahistórico a constantes antropológicas. Es cierto que, por ejemplo, Spanhel hace honor al concepto de Loch a la luz de las crisis actuales de la modernidad (1988: 7 y ss.). Sin embargo, esto no puede llenar el vacío socio-teórico de la teoría. La "presión de biografización» que pesa sobre los individuos en las sociedades modernas tardías (véase Fuchs, 1983: 366) no puede eliminarse con «competencias curriculares» ahistóricas. Los desarrollos biográficos se modifican con las «tareas de aprendizaje» históricamente modificadas. En el contexto de las biografías concretas, la estructura y la acción emergente no constituyen una «armonía preestablecida», sino un campo de tensión que influye

7 Por ello, resulta sorprendente que Loch se refiera de manera sumamente escasa al estructuralismo genético de Piaget. 
y altera la biografía. La concepción de Lochs es interesante porque logra de manera convincente vincular la cuestión biográfica con las teorías pedagógicas (véase Kaltschmid ,1988: 106 y ss.). Para la práctica formativa, por otro lado, su valor de utilidad es escaso ${ }^{8}$, puesto que no proporciona respuestas convincentes a las crisis sociales actuales y a las consecuencias de la modernización que intervienen en las biografías.

\section{El «planteamiento compensatorio»}

Precisamente esto, sin embargo, es lo que reclaman las concepciones que se han especializado en situaciones de crisis biográficas. Parece justificado caracterizarlas con el término genérico «compensatorias» porque, al menos indirectamente, están relacionado con el tratamiento de situaciones problemáticas en la educación de adultos y la eliminación de dificultades en los procesos formativos. Un paradigma que parece particularmente prometedor para acceder a las crisis biográficas es la concepción de critical life events (Filipp (ed) 1981). La identificación de tales eventos con valor tematizador no es aparentemente problemática (cf. Breloer, 1984; Siebert, 1984; Kade, 1985). Coinciden con situaciones de riesgo, especialmente en los pasajes de estatus social, p. ej., en la transición del sistema educativo al sistema laboral educación al del empleo, en el reingreso en la profesión o en el umbral de la jubilación.

La plausibilidad aparente de este acceso pedagógico a la biografía se torna problemática en una consideración más detallada. El fenómeno de los mismos «eventos críticos de la vida» y en particular el modo y manera en que se procesan individualmente en absoluto son tan claros como sugieren las «listas de eventos». Dejando de lado los pasajes de estatus sociales, relativamente estandarizados, cualquier cantidad de eventos puede convertirse en critical life events desde un punto de vista individual. Esto va desde una vergüenza embarazosa sobre el nacimiento del primer hijo hasta el accidente automovilístico trascendental. El problema no es en primera instancia la multiplicidad teóricamente concebible de las constelaciones de eventos, sino el hecho de que cada evento puede tener consecuencias completamente diferentes en diferentes biografías. Así, mientras que el nacimiento de un niño en el curso de vida de una mujer joven orientada profesionalmente puede causar una revisión del plan de vida dramática y ser solo introducida con una gran resistencia interna, en la biografía de unos «padres tardíos» en una situación acomodada, por el contrario, puede representar el descubrimiento de una vida satisfactoria apenas esperada.

Los eventos vitales críticos alcanzan a todas las formas específicas de acumulación de experiencia biográfica y a las estructuras actualmente dominantes de los procesos del curso de vida (Schütze, 1981; 1984). Son de una importancia extraordinaria si afectan a las biografías en una fase caracterizada por una gran autonomía de acción del sujeto de la biografía, o si ingresan en fases de la vida caracterizadas por una pérdida del control biográfico y complicaciones semejantes en las trayectorias (Schütze, 1981: 88 y ss.; Alheit 1984a, parte II, 37 y ss.). Su superación también depende del entorno del mundo de la vida del actor biográfico, de la funcionalidad del medio familiar o social al que pertenece ${ }^{9}$. Esto significa que no es el evento en sí mismo lo decisivo, sino la biografía a la que afecta. Para utilizar la concepción del critical-lifeevent de una manera pedagógicamente fundada se precisan o bien sólidas experiencias empíricas con los decursos biográficos de ciertos grupos de destinatarios o bien una teoría marco biográfica convincente, en la que la ambivalencia de los acontecimientos críticos de la vida pueda incluso incorporarse de manera significativa.

\footnotetext{
8 En este punto, las referencias a los estudios de Kohlberg (por ejemplo, Lempert, 1990; Hoff, 1990) serían mucho más interesantes, también para una formación continua orientada biográficamente.

9 Cf. en este punto también las objeciones críticas en la obra de Siebert 1985: 44 y s.
} 
La pedagoga de discapacitados Erika Schuchardt ha emprendido con su análisis de las biografías de discapacidad un intento de esbozar, mediante un ejemplo expuesto de manera comparativa, el proceso de un «tratamiento de la crisis» gradual de una critical life event relevante para los pacientes y sus cuidadores ${ }^{10}$. Con ello logra crear una teoría del aprendizaje de la aceptación, fundamentada empíricamente, en el caso de las personas discapacitadas y conseguir un logro (o recuperarlo) de autonomía de acción. El proceso de aprendizaje desde la realización cognitiva, pasando por la aceptación emocional, hasta la participación activa se puede fundamentar de manera tan plausible porque al mismo tiempo se puede describir como un «proceso de curación». La intervención compensatorio-pedagógica se orienta según el paradigma terapéutico. Sin embargo, con la generalización de este modelo para la educación de adultos (Schuchardt, 1993) se genera el dilema de una difusión de los conceptos «terapia», «investigación» y «formación». Schuchardt aboga enfáticamente por una superación de la separación. El umbral entre el «aprendizaje» y la «curación» se convierte así en una problemática zona gris. Una «formación de personas adultas orientada biográficamente» desaparece, por así decirlo, detrás de una configuración terapéutica que se extiende en exceso hasta la pérdida de sus contornos.

Algunos educadores de adultos muestran también tendencias similares ${ }^{11}$ cuando se refieren al concepto de terapia «amplio» de la llamada «sociología clínica» de Ulrich Oevermann (Oevermann, 1981). Los empujes continuos de la modernización hacen que, después de su aceptación, la adquisición de un sentido personal se convierta en un problema fundamental. El sentido se adquiere cada vez menos por la participación incuestionada de los individuos en la cultura y la sociedad; su generación queda en manos de los sujetos a los que estructuralmente se les exige demasiado. En este proceso, no se generan déficits de integridad de manera exclusivamente «patológica», sino también de un modo «normal». La exigencia de una acción cuasiterapéutica de instancias profesionales de tratamiento de los problemas aumenta drásticamente. Y no solo se refiere a la cura de «patologías» en el sentido clínico. La educación de adultos también asume funciones «terapéuticas» (Koring, 1987).

Detrás de este constructo de legitimación teórico-profesional inicialmente comprensible, hay, sin embargo, aporías que hay que tomar en serio. Por ello, en este punto debe ser replanteada la objeción radical del análisis del discurso (post)estructuralista: el tratamiento profesional de los déficits de significado «modernos» solo aceleraría el proceso de decadencia de los recursos de sentido tradicionales (por ejemplo, Foucault, 1971; 1974). Para la educación de adultos, es más importante señalar que una universalización de los déficits de integridad o de identidad pone en riesgo de perder el concreto establecimiento de objetivos de la acción cuasiterapéutica. Déficits de sentido subjetivo generados socialmente no pueden ser manejados individualmente; «la pérdida de mundos sociales significativamente integrados no solo afecta al "cliente", sino también al profesional que se convierte él mismo en "cliente"» (Gildemeister \& Robert, 1986). No resulta muy comprensible por qué deben escapar al proceso cuasiterapéutico de pérdida de sentido y, por esta razón, las interpretaciones representativas de las personas que están en la práctica profesional de la formación están estructuralmente menos dañadas que las de la gente común.

Ante todo, parece sintomático que la suposición de las crisis de identidad como una situación normal haya revelado hace mucho tiempo la autonomía de la dimensión biográfica. Pero nuestras vidas no consisten solo en autointerpretaciones fallidas o exitosas, sino también tenemos que lidiar con una cadena de

10 Cf. el «modelo en fases en espiral» desarrollado por esta autora en: Schuchardt, 1980: 113.

11 En este punto debe hacerse referencia, en particular, al trabajo de Schmitz (1983, 1984) y Koring (1987). 
eventos que nos ocurren. Ciertamente, hay que encajar cada vivencia en nuestro edificio experiencial, y eso significa también: ser subjetivamente «interpretado», pero sigue siendo parte de una historia recordable de manera narrativa que, junto con otras experiencias, constituye el carácter específico de nuestras vidas. Este potencial emergente de la biografía es subestimado cuando los cursos de vida son considerados solo a la luz de los déficits de integridad resarcibles cuasi-terapéuticamente (cf. Alheit, 1988). Los planteamientos compensatorios de la educación de adultos orientada a la biografía permanecen encadenados a una «construcción de normalidad» artificial. Conciben los procesos de aprendizaje sustancialmente como procesos de adaptación dirigidos. La subjetividad como un potencial emergente para la acción, que podría contrarrestar activamente los procesos sociales de deterioro, ya no está en el campo de visión. La biografía es concebida - al menos en el caso de las más dañadas- como el resultado de desarrollos «modernos», ya no como un proceso auténtico de transición, más como una «respuesta» individual a los desafíos de la modernidad tardía.

\section{El «planteamiento autobiográfico»}

Al menos en su pretensión, esta opción está conectada con concepciones que se basan en recuerdos autobiográficos. La variante metódicamente más avanzada de tal planteamiento, que ha sido probada en la educación de adultos de múltiples maneras, especialmente en los Estados Unidos, es sin duda la técnica de la «guided autobiography», desarrollada por James E. Birren (Birren \& Hedlund, 1987; Mader, 1987) ${ }^{12}$. Una característica esencial de esta propuesta es una «topical approach», una reconstrucción temáticamente guiada de la biografía. Con la ayuda de un número limitado de temas «generativos» (por ejemplo, «familia», «muerte», «cuerpo», «dinero», «tiempo», etc.), las experiencias autobiográficas son recordadas escritas, intercambiadas y procesadas, primero individualmente en un período más largo y después en pequeños grupos y en discusiones plenarias (cf. Mader 1987, :6 y ss.).

A diferencia de las concepciones debatidas hasta ahora de la formación (de personas adultas) orientada biográficamente, no solo se pone en el centro expresamente la perspectiva formativa; el procedimiento metódico y los principios didácticos también son transparentes y están bien fundamentados ${ }^{13}$ : cada tema se toma en serio y constituye una unidad de aprendizaje en sí misma («elemento temático») $)^{14}$. Los destinatarios son animados a poner por escrito sus pensamientos sobre el tema respectivo de forma concisa («elemento escrito»), un procedimiento que contribuye a la objetivación de la experiencia individual y promueve un ulterior principio didáctico, a saber, la obligación de reflexión de cada individuo («elemento singular-reflexivo»). Este confrontación individual se complementa con el intercambio de recuerdos autobiográficos singulares con otras personas en un grupo pequeño («elemento de comunicación social»). El objetivo final es el intento de una generalización tan formativa como sea posible de una experiencia de vida personal («elemento metafórico»).

A pesar de que la aproximación a la autobiografía resulte tan plausible metódicamente y exitosa evidentemente en la práctica, la concepción de Birren plantea dos cuestiones esenciales no criticadas hasta ahora. También el topical approach -y con ello la concepción del critical-life-event empleada- supone un acce-

\footnotetext{
12 La concepción de Birren se sitúa en el contexto de su investigación sobre el envejecimiento (de manera representativa: Schroots y Birren, 1988) y se refiere a un campo de trabajo nuevo y en expansión de la educación de adultos: la temática del envejecimiento y el aprendizaje, un campo de problemas que indudablemente marca la tendencia que se reforzará en la orientacion biográfica temas y métodos (cf. Mader, 1987).

13 Cf. sobre ello, una descripción detallada en Mader 1987: 13-21.

14 Sin embargo, se debe enfatizar una vez más que estos no son temas arbitrarios, sino «existenciales». El envejecimiento, la sexualidad, la muerte, etc. son cuestiones que nadie puede soslayar, y para las cuales inevitablemente debemos desarrollar una referencia autobiográfica (Mader, 1987).
} 
so cuasi «digital» a la biografía. Ello implica implícitamente que cada biografía está constituida por «topoi» existenciales de una dignidad casi antropológica. Es difícil negar que temas como la familia, el envejecimiento, la sexualidad o la muerte pertenecen al inventario de la vida humana. No cambian con las modas. Y, sin embargo, esta constatación es vigente solo en un nivel de abstracción supremo. Precisamente la dramática disminución de las fuentes de significado en el curso de los procesos de modernización actuales ha alcanzado ya el horizonte de asociación metafórica de tales «universales» culturales: lo que significan biográficamente en realidad la «familia», el «sexo» el «dinero» o la «muerte» depende de la pluralización y la etnicización de los mundos de vida y de los estilos de vida, así como también ciertamente de los marcos sociales de la biografía. Un discurso exitoso sobre tales topoi, como al que aspira la guided autobiography, depende de la proximidad del espacio social. Y esto está postulado de manera indudablemente irreflexiva en la disposición de la esta concepción. El «elemento escrito» como competencia esencial de la objetivación de la experiencia autobiográfica es altamente selectivo desde la perspectiva social. El oculto efecto creaming ${ }^{15}$ de la concepción deja aquí su huella de manera más clara. Con la guided autobiography, que por supuesto está diseñada como un proceso de aprendizaje, no como una intervención terapéutica, también acaece la estabilización (típica de la clase media) de las expectativas biográficas de la normalidad.

La segunda afinidad con los enfoques compensatorios consiste en la preferencia de las reconstrucciones biográficas interpretativas. Lo «temático», lo «escrito», y también el «elemento metafórico» fuerzan el discurso orientado biográficamente sobre las estructuras de significado de mayor predicamento de la autobiografía: sobre evaluaciones de los topoi o condensaciones metafóricas. Al hacerlo, inevitablemente se vuelve sobre la referencia a eventos o acciones de la vivencia biográfica, que permanecen vivas en las recapitulaciones narrativas (cf., Schütze, 1984; Alheit, 1984a). También aquí está fuera de toda duda que la interpretación retrospectiva de la autobiografía puede ser extraordinariamente efectiva para los procesos de aprendizaje de las personas adultas. Tales «imágenes vitales» garantizan la continuidad y consistencia de la propia vida. Sin embargo, existe el peligro de que se conviertan en meras ilusiones vitales, que la interpretación retrospectiva se desacople de las estructuras procesuales del curso de la vida y la «biografía» pierda también su dimensión de acción emergente ${ }^{16}$. La guided autobiography puede ciertamente estimular la discusión de las construcciones de la vida; la acción biográfica, por el contrario, influye en ella en todo caso indirectamente.

El plano de la acción autobiográfica fue objeto de interés en un proyecto en ciencias de la educación en el que tomó parte un grupo de destacados pedagogos de la Universidad de Bielefeld en Alemania (cf. como ejemplo: Baacke \& Schulze (eds.) 1979, 1985). Aunque trabajó expresamente de manera interdisciplinar (Baacke, 1985), su preocupación específica fue, sin duda, la «investigación biográfica pedagógica». Con ello se trataría de una reconstrucción y una ampliación de los instrumentos de las ciencias de la educación sobre diversas formas de autotematización y autoexposición de la biografía propia (Baacke, 1985: 12 y ss.; Schulze, 1985: 31 ss.). «De las historias» debe aprenderse (Baacke y Schulze (eds.) 1979), porque las historias están cerca de la acción y de los acontecimientos y pueden transformar las ideas abstractas en procesos educativos prácticos (Baacke, 1985: 13).

En este sentido concreto, aprendizaje autobiográfico significa el descubrimiento del paisaje «biografía», que ha superado la identidad del yo que hasta entonces permanecía incuestionable, tal vez de manera ingenua, o que se ha tornado temporalmente problemática: distintas historias experienciales, la peculiari-

15 El efecto creaming se refiere en este caso a una selección subrepticia de los participantes a partir de la práctica de la escritura autobiográfica. [N. trad.]

16 Cf. aquí la crítica provocativa en el polémico ensayo de Pierre Bourdieu La ilusión biográfica (1990). 
dad de los mundos de vida circundantes, rupturas y transiciones, situaciones y constalaciones como punto de partida de los desarrollos, caminos y vías de sentido único, proyectos de futuro (Schulze, 1985: 42 y ss.). El carácter autónomo de este enfoque consiste en que la biografía es considerada como un proceso multifacético y no solo como un resultado. El objetivo no es principalmente la interpretación de la historia de la vida, sino más bien su reconstrucción. Esta concepción se aproxima, aunque no ciertamente en lo metódico, al nivel de la guided autobiography. Tiene más bien carácter programático. Sin embargo, podría ser un interesante complemento a la aproximación de James E. Birren: lo particular de la biografía individual no se ha de observar solo en la historia de vida como una específica «estructuración temática» (Thomae, 1968: 329 y ss.), sino en las historias de vida en cuanto recordables narrativamente.

Este alegato de una «pedagogía narrativa» tiene ciertamente un trasfondo teórico: Con mucho, la mayor parte de nuestras acciones que son biográficamente significativas no tiene un marco de orientación de alcance biográfico general, sino más bien una especie de «concepto secular del "mundo" en el sentido del mundo cotidiano o del mundo de vida» (Habermas, 1981, II: 206). Este concepción no requiere interpretaciones altamente predictivas, pero presupone que podemos ubicarnos en espacios sociales específicos y reconocer una relación con los tiempos históricos en los que vivimos. Ambas cosas ocurren cuando nos volvemos a nuestra propia historia con un interés narrativo. «La práctica narrativa [...] sirve no solo a las necesidades triviales de comprensión de las personas con las que nos relacionamos, con las que tenemos que coordinar el trabajo conjunto; también tiene una función para la autocomprensión de las personas que necesitan objetivar su adscripción al mundo de la vida al que pertenecen en su papel actual como participantes en la comunicación. Es decir, solo pueden constituir una identidad personal cuando reconocen que la secuencia de sus propias acciones proporciona una historia de vida que se puede exponer de manera narrativa, y solo pueden formar una identidad social cuando reconocen que, más allá de la participación en las interacciones, mantienen su adscripción a los grupos sociales y, con ello, que están enredados en la historia que se puede exponer de manera narrativa de los grupos sociales. Los colectivos conservan su identidad solo en la medida en que las ideas que las personas que pertenecen a los grupos hacen de su mundo de la vida se superponen lo suficiente y se condensan en convicciones de fondo no problemáticas». (Habermas, 1981, II: 206).

Para la pedagogía, estas consideraciones teóricas tienen consecuencias. Obviamente, el desarrollo de la identidad personal se basa menos en la oferta de las identidades «representativas» que en la recapitulación narrativa de la propia vivencia autobiográfica. Esta no consiste en una ingenuamente enfática valorización de la narración en los procesos de aprendizaje biográficos, sino en el significado estructural de la narratividad para ilustración de la acción biográfica: «En la gramática de las narraciones se puede leer como nosotros identificamos y describimos los estados y eventos que se producen en un mundo de vida; cómo conectamos y secuenciamos las interacciones de los miembros de los grupos desde espacios sociales y épocas históricas hasta unidades complejas; cómo explicamos las acciones de los individuos y los eventos que les suceden, cómo explicamos las acciones de los colectivos y los destinos que soportan desde la perspectiva de la manera como hacen frente a las situaciones. Con la forma del relato elegimos una perspectiva que nos obliga «gramáticamente» a tomar como fundamento de la descripción un concepto cotidiano del mundo de vida en cuanto sistema de referencia cognitivo» (Habermas, 1981, II: 207). Precisamente nuestro saber biográfico intuitivo -aquella «gramática de narrativas»- dispone, por tanto, de contextos de referencia que pueden ser explicados en el proceso pedagógico. Al hacerlo, no solo comenzamos a comprendernos a nosotros mismos, sino también a comprender mejor las condiciones de reproducción de nuestro mundo de vida. 
Aumentamos nuestra autonomía de acción biográfica. -Parece estimulante combinar estas consideraciones sobre la narratividad autobiográfica con las experiencias metódicas de la guided autobiography-.

\section{El «planteamiento histórico»}

La gramática de las narraciones «entrelaza» a los individuos, como bien formula Habermas (1981, II: 206), también en la «historia de colectivos». El recurso autobiográfico necesariamente toca el aspecto de la identidad social. De las narraciones biográficas no aprendemos únicamente para nosotros mismos y para los mundos a los que pertenecemos; adquirimos ideas sobre la cultura, la sociedad y la historia ${ }^{17}$. Por lo tanto, tiene sentido analizar en este contexto aquellas aproximaciones que desean iniciar procesos de aprendizaje con una referencia explícita a la «historia oral» (cf., por ejemplo, Günther et al., 1985).

En tales concepciones, sin embargo, la relación entre el aprendizaje y la vida no está asociada directamente, sino con una interesante refracción: mediante las historias de vida, la historia debe hacer más trasparentes las estructuras sociales y abstractas en las constelaciones individuales. La ocasión para este modo de ver suele ser un argumento bastante didáctico: «Uno ya no se pone fácilmente en el lugar del pupilo de Dios o del espíritu del mundo; es más difícil ponerse en la posición de los poderosos y analizar los problemas sociales desde arriba como cuestiones de orden, dominación o integración. Comenzamos, más bien, a interesarnos por nosotros mismos y por el origen de nuestras propias condiciones de vida, modos de comportamiento, patrones interpretativos y posibilidades de acción: ¿Cómo se han inscrito, por ejemplo, las normas de rendimiento en nuestros cuerpos? ¿Qué relaciones de trabajo y propiedad han producido qué constelaciones familiares? ¿Qué cambios en el comportamiento y en el pensamiento han obligado al tránsito del campo a la ciudad? ¿Qué esperanzas ha destruido el fascismo? En esta documentación de lo cotidiano, cuya historia ya exterior solo puede ser aprovechada con imaginación metódica, se pregunta por la subjetividad de aquellos a quienes hemos aprendido a ver como objetos de la historia, de acuerdo con sus experiencias, sus deseos, su fuerza de resistencia, su capacidad creativa, sus sufrimientos.» (Niethammer, 1980: 9).

La biografía es en concepciones de este estilo no un lugar de aprendizaje primario; las experiencias biográficas resultan más bien en contenidos formativos. Por ello, los accesos son también posibles indirectamente -más allá de las autobiografías históricas o literarias-. Sin embargo, sigue siendo característico el contacto directo, el interrogatorio de testigos presenciales en cuanto «historia oral» autopercibida. Aquí se vuelven borrosas las fronteras entre la formación política y la «historia cotidiana» (Lüdtke, (ed) 1989), entre la formación laboral y la «historia desde abajo» (Alheit \& Wollenberg, 1982: 274 y ss.). La «biografía pedagógica» se convierte en el medio de formación de la identidad social, política y cultural. No cabe duda de que el peligro de el «planteamiento histórico» radica en la problemática distancia de las experiencias biográficas de las personas que están aprendiendo, posiblemente incluso en la formación de la tradición y la iconografía acríticas ${ }^{18}$. No obstante, esto no puede relativizar la posibilidad de que, con la «visión histórica», quede abismado un aspecto esencial del recuerdo autobiográfico: la comprensión de la interconexión del propio destino con la historia de una cultura social concreta.

El principio del gräv där du står («excava donde estés parado») - presentado por el escritor sueco Sven Lindqvist a fines de la década de los setenta en un libro inusualmente popular (Lindqvist, 1989 [1978])-

\footnotetext{
17 Aquí se puede señalar la elevada relevancia metodológica de la narratividad en la historiografía, que indudablemente tiene desde la importante obra de Arthur C. Danto, Analytical Philosopby of History (cf. sobre este asunto, de manera detallada, Rossi (ed.) 1987).

18 Para una exposición detallada de la crítica a este riesgo, cf. Alheit \& Dausien, 1990
} 
ha llevado a uno de los mayores movimientos de educación laica en Europa. Solo en Suecia están activos casi 10.000 círculos de estudio (Dammeyer, 1989: 293 y s.). Y una parte considerable de su trabajo consiste en investigaciones de historia oral. La motivación de las personas que están aprendiendo sería la convicción de que «el futuro... comenzó ayer» y de que se entienderían a sí mismos y a sus propias vidas solo si comprenden su pasado.

Ciertamente, parece justificado en concepciones equiparables la cuestión de si puede discutirse todavía con sentido de la formación (de personas adultas) orientada biográficamente. Por otro lado, esta puntualización también se puede invertir radicalmente: ¿podemos tener «hecha», en sentido práctico, una historia que está en gran parte usurpada por las interpretaciones de poderosas organizaciones y grupos de interés, que incluso se superponen (ya en Benjamin, 1965), y en general si seguimos la vía de prescindir de los seres humanos -aunque «no por propia voluntad»— (Marx/Luxemburg)? ¿No es inevitable el «desvío» por las historias de vida concretas si queremos entender el pasado? Serían comprensibles, por ejemplo, el trauma de la Guerra de Vietnam para la sociedad de los Estados Unidos o las experiencias contradictorias de la «reunificación» en Alemania? -Los procesos de aprendizaje orientados a la biografía parecen fertilizar no solo la pedagogía sino también la historiografía misma-.

\section{El «planteamiento intercultural»}

El hecho de que la ilustración del saber biográfico produzca referencias extraordinariamente cercanas a los desarrollos en la sociedad como un todo puede ser corroborado por otro concepto pedagógico. La percepción de la «extranjeridad» de las personas migrantes como problema social o, a la inversa, el redescubrimiento de la «etnicidad» como protección de su estabilidad psicológica (ya en Klemm, 1985) han hecho que los procesos educativos orientados biográficamente sean inusualmente populares en la llamada «educación intercultural». ${ }^{19}$

No obstante, la concepción básica de esta aproximación es tan simple como problemática. Se basa en una «hipótesis de la diferenciación de la modernidad» (Bukow \& Llaryora, 1988): migrantes pasan su biografía presuntamente en dos «culturas» incompatibles, la cultura de sus «países de acogida» y la cultura de procedencia. La cultura de origen generalmente se identifica según los tipos ideales con los patrones tradicionales de estilos de vida identificados y folclorísticamente sobredimensionados. La cultura del país de destino se considera incuestionablemente «moderna» y está determinada por orientaciones axiológicas racionales y universalistas. El foco de una cultura es la familia, el centro de la otra es la profesión. La coerción de oscilar entre ambas culturas crea problemas de identidad e integración y precisa asistencia pedagógica. Con ello hay un significado secundario sobre si, en cuanto meta de la pedagogía intercultural, se requiere una segunda infancia, por así decir «moderna», como un objetivo de la educación intercultural (Bukow \& Llaryora, 1988: 15), o si parece más valiosos un «fortalecimiento del sentido de pertenencia a la propia minoría» (Klemm, 1985: 181). Como resultado se genera una «trampa de la modernización» (Apitzsch, 1990: 15), que sobreestiliza didácticamente la peculiaridad étnica construida para justificar las intervenciones educativas (Hamburger, 1988). Aquí observamos un paralelo interesante con los «planteamientos compensatorios», ya discutidos críticamente.

En el caso de tales diferencias construidas de modernidad se ignoran los reales problemas de modernización, lo que puede suceder muy fácilmente desde la perspectiva biográfica con las personas migrantes.

19 Es notable que la destacada investigadora de biografía y migración Ursula Apitzsch se haya enfrentado críticamente desde el principio con este hecho (véase Apitzsch, 1989a; 1989b; 1990a; 1990b). 
Como regla general, las culturas de sus territorios no son de ninguna manera «premodernas». A menudo, la decisión migratoria es el resultado de un enfrentamiento crítico con los procesos de modernización en la propia sociedad de origen. El proceso migratorio se plantea no como el camino de un mundo tradicional no fragmentado hacia un entorno moderno problemático, sino como un proceso planificado de «modernización individual» (Inkeles, 1984: 373 y ss.) con una orientación clara hacia las agencias de modernización, la escuela y las empresas industriales, en la sociedad de acogida (Apitzsch, 1990: 15 y s.). El problema de esta amplia expectativa no es solo el riesgo de desintegración social, sino el riesgo de una trayectoria biográfica negativa con una dinámica considerable (Alheit, 1984b) ${ }^{20}$.

Los resultados de los procesos de modernización individuales comparables son en unos casos positivos, con conexiones asombrosamente no problemáticas, más bien convencionales (relativas a la familia) con orientaciones claramente modernas (relativas a la profesión), especialmente en mujeres; pero en otros casos negativos, a menudo en los miembros masculinos de las generaciones posteriores, con el colapso de los recursos de planificación biográfica, porque el retorno a la ethnicity ya no es una salida real (Apitzsch 1989a; 1989b). Esta observación sugiere que las biografías de personas migrantes «no deberían interpretarse culturalísticamente en contextos étnicos, sino que más bien las dificultades de los tránsitos de estatus atraviesan hoy la sociedad multicultural, que son un problema de la modernización de esta sociedad, que los grupos de migrantes no experimentan más tarde, sino antes y de una manera más radicalmente que otros grupos de la sociedad de acogida» (Apitzsch, 1990a: 18).

Este ejemplo deja claro cuán problemáticos serían los constructos normativos de procesos o las identidades biográficas para una concepción pedagógica. Por el momento, los profesionales de la educación están extraordinariamente mal preparados para acompañar procesos de aprendizaje intercultural orientados a la biografía. Los considerables riesgos, pero también las oportunidades, de las biografías migratorias modernas son, en cierto sentido, «anticipaciones» de opciones sociales universalizadas en las biografías modernas. Por lo tanto, representan importantes recursos -tanto positivos como negativos- para una pedagogía cuyo objetivo es la «biograficidad», la producción de autonomía individual de acción en el curso de vida ${ }^{21}$.

\section{El planteamiento emancipatorio}

La experiencia estructural de la incertidumbre de los tiempos biográficos, de la individualización y la marginalización es también el fundamento de concepciones formativas que pueden ser caracterizados razonablemente por el término «emancipadoras» porque explícitamente abogan por una transformación de aquellas estructuras básicas que permiten el despliegue de posibilidades biográficas, notoriamente obstaculizadas, en grupos particulares de la sociedad. Esto es particularmente cierto de los planteamientos biográficos del trabajo formativo feminista. Resulta paradigmático en Schmeling (1988). Ella descubre la biografía femenina como un «campo de aprendizaje político», porque es precisamente la «biografía normal» sugerida socialmente la que promueve la visión de la desigualdad estructural y habitual en la vida de los sexos: el efecto «gender-doing» del sistema educativo (Rabe-Kleberg, 1986; 1987; 1988b) y la intensificación de este efecto en las transiciones al sistema ocupacional.

20 Precisamente este dilema biográfico puede convertirse en una «trampa» para los miembros de la segunda y la tercera generación de inmigrantes. Aquí, las esperanzas de carreras formativas y profesionales están aún más extendidas que en la primera generación de migrantes; y el retorno sin problemas a la colonia étnica está bloqueado en la mayor parte de los casos (véanse los interesantes resultados de la investigación sobre biografías de aprendizaje de biografías de mujeres miembros de las generaciones posteriores en Apitzsch, 1989b; 1990a; 1990b).

21 En este contexto, aparece por primera vez el concepto principal del presente estudio. Aún no representa un concepción o incluso una teoría elaborada. Es como si fuera un «concepto programático»; anuncia una estrategia educativa. 
Por regla general, las mujeres -biográficamente- no reciben los mismos recursos de planificación por parte del sistema educativo que los hombres. Se les niega la preparación sistemática en las estrategias de acción biográfica y las capacidades de control que median en passant el inventario estándar de la socialización masculina. Por lo tanto, es más probable que las mujeres caigan en «trampas formativas» y que tengan más probabilidades de convertirse en perdedoras en el proceso de modernización del «capital humano» (Rabe-Kleberg, 1988a; 1988b). La causa de estas trampas estructurales es el patrón básico de una «doble socialización» (Becker-Schmidt, 1987; 1996), es decir, la integración escindida de las mujeres en el trabajo remunerado y en el familiar. Este modo de socialización -relativamente independiente de las características estructurales sociales- parece influir, con asombrosa persistencia, en la «lógica» de la ubicación social, la ubicación en el proceso de trabajo remunerado y en el aparato de dominación social. Resulta interesante, sin embargo, que este «patrón» no produce ningún resultado definitivo, sino más bien la pluralidad biográfica de las situaciones de vida de las mujeres (paradigmáticamente Moen, 1985; Dausien, et al., 1990).

Alice Rühle-Gerstel (1932) ya llamó la atención sobre que de ello se deducía, por así decir, una perspectiva «natural» del trabajo de formación de las mujeres con orientación biográfica -que se vuelve críticamente contra el movimiento burgués de mujeres-. Ella argumenta convincentemente, «que no se trata de realizarse a sí misma, como si en cada mujer hubiera una sustancia antecedente, fácilmente identificable, que garantizara la unidad y la progresión del conocimiento. El problema ha de desarrollarse en otro nivel teórico: no disolver diferencias y límites, sino reconocerlos. En el camino que parte de símismo hay realidades que hay que perdibir y realizar, que no se encuentran en las proximidades.» (cit. según Nordmann, 1988: 121). El objetivo y el asunto político son, al mismo tiempo, la superación de cada constelación biográfica individual, el camino «que parte de sí mismo». Pero eso significa nada menos que subjetivismo. Para la formación de las mujeres, puede convertirse en una nueva forma de aprendizaje enérgico, una especie de movimiento social (Schiersmann, 1983). La resistencia a las trampas impuestas de la estructura biográfica no solo cambia los cursos de vida individuales, sino que también afecta la organización social del trabajo y la formación en general.

Por lo demás, parece sintomático que, como ejemplo de procesos formativos emancipatorios, ya no se ofrezcan concepciones de los movimientos sociales tradicionales ${ }^{22}$, sino ideas de contextos feministas. Este hecho ya no puede explicarse mediante concesiones voluntarias al espíritu de la época. La característica de las planteamientos emancipatorios en la formación de las mujeres consiste precisamente en el hecho de que la experiencia biográfica se toma en serio como punto de partida de los procesos formativos no solo en su peculiaridad subjetiva, sino que también entra lo «privado» en un proceso de politización ${ }^{23}$. Con ello, no están excluidas formas colectivas de resistencia, sino que están expresamente previstas (Schmeling, 1988: 118 y ss.). Sin embargo, se trata de «nuevas asociaciones» (Alheit, et al., (eds.) 1990, II: 913 y ss.), que no se condensan «por crecimiento natural» en agregados sociales colectivos, sino por medio de una decisión libre y consciente de los individuos involucrados en el movimiento social. El proceso de aprendizaje político también parece cambiar su rostro; incluso el aprendizaje colectivo tiene que comenzar con la biografía concreta.

22 Esto ciertamente no significa que la orientación biográfica no tenga cabida, por ejemplo, en la formación sindical. Todo lo contrario: el cultivo de «nuevos modelos culturales», especialmente entre los trabajadores jóvenes (Zoll, et al., 1989), puede requerir conceptos formativos más sensibles con el curso de la vida, que incorporen y amplíen las ideas del «enfoque experiencial» basado en la teoría en Oskar Negt (1971) (cf. Alheit \& Wollenberg ,1982). Nadie, por supuesto, puede afirmar que el empuje para una mayor sensibilidad a las biografías como tema del trabajo político de formación haya partido del movimiento obrero y sindical actual. En este punto, el movimiento de mujeres es indiscutiblemente el protagonista y, al mismo tiempo, el portador de la esperanza al mismo tiempo.

23 El célebre eslogan feminista «iLo privado es político.» se refiere también a la comprensión de que las estructuras sociales de dominio y sus condiciones de reproducción no dejan intacta la privacidad. Por ello, por supuesto, las mujeres son las más afectadas (véase Schmeling, 1988: 71 y ss.). 


\section{Conclusión}

El resultado más interesante de la revisión crítica de diferentes plantemientos del trabajo formativo orientado biográficamente no es la complejidad contradictoria de las referencias teóricas. El hallazgo más asombroso es, sin duda, el amplio rango de las concepciones que cubren las concepciones formativas orientadas biográficamente: la amplitud de los planteamientos va desde intervenciones terapéuticas individuales hasta el compromiso sociopolítico, desde temas clásicos de la formación continua de índole general y sociocultural hasta las nuevas cuestiones de formación continua profesional y política. Por lo tanto, la orientación de la biografía en la formación de personas (adultas) puede considerarse como una especie de «paradigma oculto» que, además, sugiere un cambio gradual en las disposiciones de los profesionales.

\section{Referencias bibliográficas}

Alheit, Peter (1984a): "Biographieforschung in der Erwachsenenbildung" (parte I) en Literaturund Forschungsreport Weiterbildung, vol. 13, 40-54; (parte II) en Literaturund Forschungsreport Weiterbildung, vol. 14, 31-67.

Alheit, Peter (1984b). Zum Problem des Einsatzes biographischer Methoden bei der Erhebung von zeitlich-diachronen Entwicklungsverläufen der beruflichen Sozialisation ausländischer Jugendlicher. Expertis für den Modellversuch «Ausbildung italienischer Jugendlicher im nationalen und binationalen Verbund». Bremen: Universität Bremen.

Alheit, Peter (1988): “Alltagszeit und Lebenszeit. Über die Anstrengung, widersprüchliche Zeiterfahrungen «in Ordnung zu bringen»" en Rainer Zoll (ed.): Zerstörung und Wiederaneignung von Zeit. Frankfurt am Main: Suhrkamp.

Alheit, Peter \& Bettina Dausien (1990): “Biographie” en Hans Jörg Sandkühler (ed.): Enąyklopädie zu Philosophie und Wissenschaften, tomo 1. Hamburg: Meiner.

Alheit, Peter \& Jörg Wollenberg (1982): “Der «Erfahrungsansatz» in der Arbeiterbildung” en Ekkehard Nuissl (ed.): Taschenbuch der Erwachsenenbildung. Baltmannsweiler: Schneider.

Alheit, Peter et al., (eds.) (1990). Bildung in der Arbeitsgesellschaft. Ergebnisse und Perspektiven des Forschungsschwerpunkts Arbeit und Bildung (parte II) (Forschungsreibe des Forschungsschwerpunkts «Arbeit und Bildung», vol. 14, Bremen: University Bremen Press.

Apitzsch, Ursula (1989a): "Interkulturelle Bildung”, Report, vol. 23, 71-81.

Apitzsch, Ursula (1989b): Normative Orientierungen italienischer Migrantenkinder zwischen Herkunftsfamilie und Beruf. En: Bildung in der Arbeitsgesellschaft. Zum Spannungsverhältnis von Arbeit und Bildung heute. Dokumentation des 10. Bremer Wissenschaftsforums vom 11. bis 13. Oktober 1988. Bremen: Universität Bremen, 228-248.

Apitzsch, Ursula (1990b). Migration und Biographie. Zur Konstitution des Interkulturellen in den Bildungsgängen junger Erwachsener der zweiten Migrantengeneration. Bremen: Habilitationsschrift Universität Bremen (manuscrito inédito).

Baacke, Dieter \& Theodor Schulze (eds.) (1979). Aus Geschichten lernen. Zur Einübung pädagogischen Verstehens. München: Juventa.

Baacke, Dieter \& Theodor Schulze (eds.) (1985). Pädagogische Biographieforschung. Orientierungen, Probleme, Beispiele. Weiheim und Basel: Beltz. 
Baacke, Dieter (1985): "Biographie: Soziale Handlung, Textstruktur und Geschichten über Identität. Zur Diskussion in der sozialwissenschaftlichen und pädagogischen Biographieforschung sowie ein Beitrag zu ihrer Weiterführung” en Dieter Baacke \& Theodor Schulze (eds.): Pädagogische Biographieforschung. Orientierungen, Probleme, Beispiele. Weiheim und Basel: Beltz.

Beck, Ulrich (1986). Risikogesellschaft. Auf dem Weg in eine andere Moderne. Frankfurt am Main: Suhrkamp.

Becker-Schmidt, Regina (1987): "Die doppelte Vergesellschaftung - die doppelte Unterdrückung: Besonderheiten der Frauenforschung in den Sozialwissenschaften” en Unterkirchen, Lilo; Wagner, Ina (Hg.): Die andere Hälfte der Gesellschaft. Österreichischer Soziologentag 1985. Wien.

Becker-Schmidt, Regina (1996): “Einheit Zweiheit Vielheit. Identitätslogische Implikationen in feministischen Emanzipationskonzepten”, Zeitschrift für Frauenforschung, vol. 14, 1/2, 5-18.

Benjamin, Walter (1965). Geschichtsphilosophische Thesen. En: Walter Benjamin, Zur Kritik der Gewalt und andere Aufsätze. Mit einem Nachwort von Herbert Marcuse. Frankfurt am Main: Suhrkamp.

Birren, James E. \& Bonnie Hedlund (1987): “Contributions of autobiography to developmental psychology" en Nancy Eisenberg (ed.): Contemporary Topics in Developmental Psychology. New York: John Wiley \& Sons.

Bourdieu, Pierre (1990): “Die biographische Illusion”, Bios, vol. 3, núm. 1, 75-81.

Breloer, Gerhard (1984): “《Kritische Lebensereignisse» - ein lebenswelt-analytischer Forschungsansatz für die Erwachsenenbildung?” en Erhard Schlutz \& Horst Siebert (eds.): Zur Identität der Erwachsenenbildung. Bremen: Bremen University Press.

Bukow, Wolf-Dietrich \& Roberto Llaryora (1988). Mitbürger aus der Fremde. Soziogenese ethnischer Minoritäten. Opladen: Westdeutscher Verlag.

Dammeyer, Martin (1989): Die Zukunft hat gestern begonnen. Nachwort zu: Sven Lindqvist, Grabe wo du stehst. Handbuch zur Erforschung der eigenen Geschichte. Bonn: J.H.W. Dietz. Dannefer, Dale \& Ralph R. Sell (1988): Age structure, the life course and, aged heterogeneity'.

Dausien, Bettina et al., (1990): "Frauenarbeit und Frauenbildung. Theoretische und empirische Befunde in der Perspektive des Geschlechterverhältnisses" en Peter Alheit et al., (eds.): Bildung in der Arbeitsgesellschaft. Ergebnisse und Perspektiven des Forschungsschwerpunkts Arbeit und Bildung (Teil II) (Forschungsreihe des Forschungsschwerpunkts “Arbeit und Bildung”, Bd. 14, Bremen: University Bremen Press.

Elder, Glen H. (1985): "Perspectives on the life course” en Glen H. Elder (ed.): Life Course Dynamics: Trajectories and Transitions. 1968-1980. Ithaka: Cornell University Press.

Filipp, Sigrun-Heide (ed.) (1981). Kritische Lebensereignisse. München, Wien, Baltimore: Urban \& Schwarzenberg.

Foucault, Michel (1971). Die Ordnung der Dinge. Frankfurt am Main: Suhrkamp.

Foucault, Michel (1974). Die Ordnung des Diskurses. Frankfurt am Main: Suhrkamp.

Fuchs, Werner (1983): “Jugendliche Statuspassage oder individualisierte Jugendbiographie?”, Soziale Welt, vol. 34, 341-371. 
Gamm, Hans-Jochen (ed.) (1984): Führung und Verfübrung. Pädagogik im Nationalsozialismus. Eine Quellensammlung. Frankfurt am Main, New York: Campus.

Gildemeister, Regine \& Günther Robert (1986): Identität als Gegenstand und Ziel psychosozialer Arbeit. Erlangen: (unveröffentlichtes Manuskript).

Günther, Elke et al., (1985): "Lebensgeschichten verstehen lernen. Ein Bericht über die Arbeit der «Berliner Projektgruppe Lehrerlebensläufe»” en Dieter Baacke \& Theodor Schulze (eds.): Pädagogische Biographieforschung. Orientierungen, Probleme, Beispiele. Weiheim und Basel: Beltz.

Habermas, Jürgen (1981). Theorie des kommunikativen Handelns, 2 tomos, Frankfurt am Main: Suhrkamp.

Hamburger, Franz (1988): "Identität und Migration" en Emigratione ed identitá culturale Emigration und kulturelle Identität. Encuentro 12.12.1988. Universität Frankfurt. Frankfurt am Main: VAS, 31-35.

Hoff, Ernst-Hartmut (1990): "Kontrolle und Moral. Problematische Arbeitsprodukte im Urteil von Arbeitern” en Felix Frey \& Ivars Udris (eds.): Das Bild der Arbeit, Bern, Stuttgart, Toronto: Huber.

Inkeles, Axel (1984): “Was heißt «individuelle Modernität»?” en Traugott Schöfthaler \& Dietrich Goldschmidt (eds.): Soziale Struktur und Vernunft. Jean Piagets Modell entwickelten Denkens in der Diskussion kulturvergleichender Forschung. Frankfurt am Main: Suhrkamp.

Kade, Jochen (1985): "Diffuse Zielgerichtetheit. Rekonstruktion einer unabgeschlossenen Bildungsbiographie” en Dieter Baacke \& Theodor Schulze (eds.): Pädagogische Biographieforschung. Orientierungen, Probleme, Beispiele. Weiheim und Basel: Beltz.

Kaltschmid, Jochen (1988): "Biographie und Pädagogik" en Andreas Kruse et al., (eds.): Gerontologie. Wissenschaftliche Erkenntnisse und Folgerungen für die Praxis. München: Bayerischer Monatsspiegel Verlagsgesellschaft.

Klemm, Klaus (1985): "Interkulturelle Erziehung - Versuch einer Eingrenzung”, Die dentsche Schule, Vol. $77,176-187$.

Kohli, Martin (1985): "Die Institutionalisierung des Lebenslaufs. Historische Befunde und theoretische Argumente”, Kölner Zeitschrift für Soziologie und Sozialpsychologie, Vol. 37, 129.

Kohli, Martin (1989): "Institutionalisierung und Individualisierung der Erwerbsbiographie. Aktuelle Veränderungstendenzen und ihre Folgen” en Dietmar Brock et al., (eds.): Subjektivität im gesellschaftlichen Wandel. München: DJI-Verlag, 249-278.

Körber, Klaus (1989): “Zur Antinomie von politisch-kultureller und arbeitsbezogener Bildung in der Erwachsenenbildung", Bildung in der Arbeitsgesellschaft. Zum Spannungsverbältnis von Arbeit und Bildung heute. Dokumentation des 10. Bremer Wissenschaftsforums vom 11. bis 13. Oktober 1988. Bremen: Universität Bremen, 126-151.

Koring, Bernhard (1987): "Erwachsenenbildung und Professionstheorie. Überlegungen im Anschluß an Oevermann” en: Klaus Harney et al., (eds.): Professionalisierung der Erwachsenenbildung. Fallstudien-Materialien-Forschungsstrategien. Frankfurt am Main, Bern, New York, Paris: Peter Lang.

Lempert, Wolfgang (1990): "Moralische Sozialisation im Beruf”, Zeitschrift für Berufsund Wirtschaftspädagogik, Vol. 86, H. 1, 3-22. 
Lindqvist, Sven (1989) [1978]. Grabe wo du stehst. Handbuch zur Erforschung der eigenen Geschichte. Bonn: J.H.W. Dietz Nachf.

Loch, Werner (1979). Lebenslauf und Eræiehung (Neue pädagogische Bemühungen, Bd. 79). Essen: Neue deutsche Schule Verlagsgesellschaft.

Loch, Werner (1981): “Anfänge der Erziehung - Zwei Kapitel aus einem verdrängten Curriculum” en Friedrich Maurer (ed.): Lebensgeschichte und Identität. Beiträge zu einer biographischen Anthropologie. Frankfurt am Main: Fischer.

Lüdtke, Alf (1989). Alltagsgeschichte. Zur Rekonstruktion historischer Erfahrungen und Lebensweisen. Frankfurt am Main, New York: Campus.

Mader, Wilhelm (1987). Autobiographie und Bildung Zur Theorie und Praxis der Guided Autobiography. Bremen: (unveröffentlichtes Manuskript).

Moen, Phyllis (1985): “Continuities and discontinuities in women's labor force activity” en Glen H. Elder (ed.): Life Course Dynamics: Trajectories and Transitions. 1968-1980. Ithaka: Cornell University Press.

Negt, Oskar (1971). Soziologische Phantasie und exemplarisches Lernen. Zur Theorie und Praxis der Arbeiterbildung, 2. überarbeitete Auflage. Frankfurt am Main: Europäische Verlagsanstalt.

Niethammer, Lutz (1980): “Einführung” en Lutz Niethammer (ed.): Lebenserfabrung und kollektives Gedächtnis. Die Praxis der «Oral History». Frankfurt am Main: Syndikat.

Nordmann, Ingeborg (1988): "Im Zick-Zack zwischen weiblichem und männlichem Entwurf”, Freibeuter, Vol. 38, 119-128.

Oevermann, Ulrich (1981). Professionalisierungstheorie. Vortragstranskript. Frankfurt am Main: (unveröffentlichtes Manuskript).

Rabe-Kleberg, Ursula (1986): “Was hat die Bildungsreform den Frauen gebracht?”, Vorgänge, vol. 81, núm. 3, 60-68.

Rabe-Kleberg, Ursula (1987): "Bildung für Frauen im Abseits? Oder: Das Abseits als unsicherer Ort. Einige Überlegungen zur Bildungsdiskussion” en Ilona Ostner (ed.): Frauen. Soziologie der Geschlechterverbältnisse (Soziologische Revue. Sonderheft 2).

Rabe-Kleberg, Ursula (1988a): "Frauenarbeit - Die andere Seite der Facharbeit. Zur Genese weiblicher Hausund männlicher Lohnarbeit" en Rainer Drechsel et al., (eds.): Berufspolitik und Gewerkschaften. Gewerkschaftliches Berufsverständnis und Entwicklung der Lohnarbeit (Forschungsreihe des Forschungsschwerpunkts «Arbeit und Bildung», Bd. 9). Bremen: Bremen University Press.

Rabe-Kleberg, Ursula (1988b): “Weibliches Arbeitsvermögen» und soziale Berufe - ein gutes Verhältnis”, Frauenforschung (Informationsdienst des Forschungsinstituts Frau und Ge sellschaft), vol. 6, núm. 4, 28-31.

Rossi, Paolo (ed.) (1987). Theorie der modernen Geschichtsschreibung. Frankfurt am Main: Suhrkamp.

Schiersmann, Christiane (1983): "Frauenbildung zwischen institutionalisierten Lernformen und sozialer Bewegung" en Erhard Schlutz (ed.): Erwachsenenbildung zwischen Schule und sozialer Arbeit. Bad Heilbrunn: Klinkhardt. 
Schleiermacher, Friedrich D. E. (1957). Pädagogische Schriften. Unter Mitarbeit von Theodor Schulze herausgegeben von Erich Weniger, vol. 1. Düsseldorf und München: Küpper.

Schmeling, Birgit (1988). Der «biographische Ansatz» in der Frauenbildungsarbeit Theoretische Voraussetzungen und praktische Erfahrungen. Bremen: Universität Bremen (tesis).

Schmitz, Enno (1983): “Zur Struktur therapeutischen, beratenden und erwachsenenpädagogischen Handelns" en Erhard Schlutz (ed.): Erwachsenenbildung zwischen Schule und sozialer Arbeit. Bad Heilbrunn: Klinkhardt.

Schmitz, Enno (1984): “Erwachsenenbildung als lebensweltbezogener Erkenntnisprozeß” en Dieter Lenzen (ed.): Enaylklopädie der Erziehungswissenschaft. Handbuch und Lexikon der Eržiehung, Bd. 11. Stuttgart: Klett-Cotta.

Schroots, Johannes J.F. \& James E. Birren (1988): “The nature of time: Implications for research on aging", Comprehensive Gerontology C, vol. 2, 1-29.

Schuchardt, Erika (1980). Soziale Integration Behinderter, 2 Teilbände. Braunschweig: Westermann.

Schuchardt, Erika (1993): “Innovative Forschung - Beispiel Biographischer Ansatz” en Hans Tietgens (ed.): Beiträge der Bezugswissenschaften zur Erwachsenenbildung (Studienbibliothek für Erwachsenenbildung, Bd. 4). Frankfurt am Main: Pädagogische Arbeitsstelle Deutscher Volkshochschulverband.

Schulze, Theodor (1985): "Lebenslauf und Lebensgeschichte. Zwei unterschiedliche Sichtweisen und Gestaltungsprinzipien biographischer Prozesse” en Dieter Baacke \& Theodor Schulze (eds.): Pädagogische Biographieforschung. Orientierungen, Probleme, Beispiele. Weiheim und Basel: Beltz.

Schütze, Fritz (1981): "Prozeßstrukturen des Lebensablaufs" en Joachim Matthes et al., (eds.): Biographie in handlungswissenschaftlicher Perspektive. Nürnberg: Verlag der Nürnberger Forschungsvereinigung e.V.

Schütze, Fritz (1984): “Kognitive Figuren des autobiographischen Stegreiferzählens” en Martin Kohle \& Günther Robert (eds.): Biographie und soziale Wirklichkeit. Neue Beiträge und Forschungsperspektiven. Stuttgart: Metzler.

Siebert, Horst (1984): “Lernen und Lebenswelt” en Erhard Schlutz \& Horst Siebert (eds.): Zur Identität der Erwachsenenbildung. Bremen: Bremen University Press.

Siebert, Horst (1985). Lernen im Lebenslauf. Zur biographischen Orientierung der Erwachsenenbildung. Frankfurt am Main: Deutscher Volkshochschul-Verband.

Spanhel, Dieter (ed.) (1988). Curriculum Vitae (Neue pädagogische Bemühungen, Bd. 98). Essen: Neue deutsche Schule Verlagsgesellschaft.

Thomae, Hans (1968). Das Individuum und seine Welt. Göttingen: Hogrefe.

Zoll, Rainer et al., (1989). «Nicht so wie unsere Eltern!» Ein neues kulturelles Modell? Opladen: Westdeutscher Verlag.

\section{Nota biográfica}

Peter Alheit ha sido catedrático en las universidades de Tübingen y Göttingen, donde regentó el Seminario Pedagógico (el más antiguo de Alemania). En la actualidad es profesor emérito. Ha sido profesor 
visitante en Santa María (Brasil), Viena, Xiamen (China) y Chulalongkorn (Tailandia). Ha publicado más de 50 libros y más de 300 artículos. Sus obras han sido traducidas a más de 13 lenguas. Ha dirigido más de 50 tesis doctorales. 